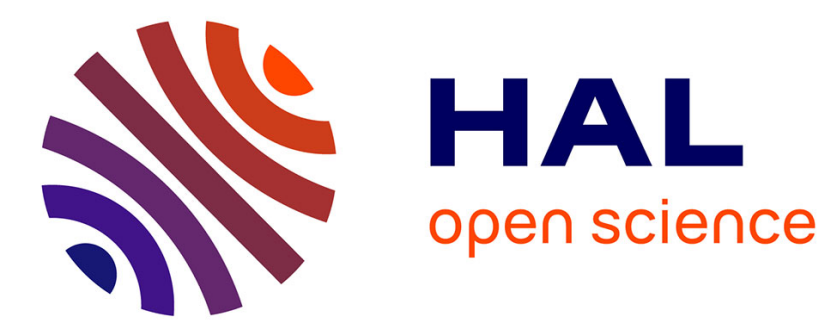

\title{
Low frequency instabilities during dust particle growth in a radio-frequency plasma
}

Maxime Mikikian, Marjorie Cavarroc, Lénaïc Couëdel, Laifa Boufendi

\section{To cite this version:}

Maxime Mikikian, Marjorie Cavarroc, Lénaïc Couëdel, Laifa Boufendi. Low frequency instabilities during dust particle growth in a radio-frequency plasma. Physics of Plasmas, 2006, 13, pp.092103. 10.1063/1.2337793 . hal-00097761

\section{HAL Id: hal-00097761 https://hal.science/hal-00097761}

Submitted on 23 Nov 2009

HAL is a multi-disciplinary open access archive for the deposit and dissemination of scientific research documents, whether they are published or not. The documents may come from teaching and research institutions in France or abroad, or from public or private research centers.
L'archive ouverte pluridisciplinaire HAL, est destinée au dépôt et à la diffusion de documents scientifiques de niveau recherche, publiés ou non, émanant des établissements d'enseignement et de recherche français ou étrangers, des laboratoires publics ou privés. 


\title{
Low frequency instabilities during dust particle growth in a radio-frequency plasma
}

\author{
Maxime Mikikian, ${ }^{*}$ Marjorie Cavarroc, Lénaïc Couëdel, and Laïfa Boufendi \\ GREMI, Groupe de Recherches sur l'Energétique des Milieux Ionisés \\ UMR6606, CNRS/Université d'Orléans, \\ 14 rue d'Issoudun, BP6744, \\ 45067 Orléans Cedex 2, France
}

\begin{abstract}
In this paper, instabilities appearing in a dusty plasma are experimentally investigated. These low frequency self-excited instabilities appear during dust particle growth and are characterized by a frequency spectrum evolving during this process. Beginning, time evolution and main characteristics of these instabilities are investigated thanks to electrical and optical measurements. Both signals show a clear evolution scheme with a well-defined succession of phases. From the beginning to the end of this scheme, regular oscillations and/or chaotic regimes are observed. Finally, instabilities stop when the dust particle size reaches few hundreds of nanometers and a stable three-dimensional dust cloud is obtained. A dust free region called void is then usually observed in the plasma center.
\end{abstract}

PACS numbers: 52.27.Lw, 52.35.-g, 52.25.Vy, 52.25.Gj, 52.35.Ra

\section{INTRODUCTION}

In a plasma, dust particles can be grown using reactive gases like silane [1-3] or by ion bombardment on materials [4-9]. This formation is actively studied due to strong consequences that can arise from the presence of dust in plasma processing reactors $[10,11]$ for microelectronics where cleanliness is a major requirement [12]. Furthermore, the interest is actually increasing due to the fact that nanometer dust particles can be useful for industrial applications like solar cells [13] or memories [14]. Laboratory dusty plasmas are also a very efficient way to produce and study astrophysical dusty media like planet atmospheres [15]. In capacitively coupled radiofrequency discharges, a dense cloud of submicron dust particles filling the whole space between the electrodes can be obtained. During their growth, dust particles acquire a negative charge by attaching more and more electrons [16-18]. Consequently, the growth can be studied through its influence on the plasma characteristics and in particular on the discharge current harmonics. Indeed, the current third harmonic is a robust tool to accuratly follow and identify growth steps [19]. Dust particle growth strongly affects plasma properties and can induce plasma instabilities. Self-excited instabilities have been observed in silane plasmas [20] (frequencies of few $\mathrm{kHz}$ for dust particles of few nanometers) and also in dusty plasmas produced by sputtering a carbon target $[7,8]$ (frequencies around $100 \mathrm{~Hz}$ for dust particles around hundred nanometers). In this last experiment, the authors describe two different instability modes called the filamentary mode and the great void mode. The first one appears approximately one minute after the plasma ignition and is characterized by a broadband spectrum centered around $100 \mathrm{~Hz}$. It corresponds to a beamlike striation of dust density and plasma glow. As dust particles are still growing, this stage is then followed by the great void mode which corresponds to the formation of a dust free region called void [7, 9, 21-23] and its ro-

*Electronic address: maxime.mikikian@univ-orleans.fr tation in a horizontal plane in between the electrodes. The void region is due to the equilibrium between various forces acting on the negatively charged dust particles. These forces are principally due to ion drag, electric fields, thermal gradients, gas flow and gravity, and they define the cloud shape. The ion drag force is presently actively studied because it is suspected to be the main responsible of the void formation [8, 24-33], pushing the dust particles away from the discharge center. In this paper, observations and analyses of instabilities appearing during dust particle growth are performed. These instabilities look like the filamentary and great void modes in some stage of their evolution. Nevertheless, their time evolution is much more complicated and seven different regimes can be identified. Indeed, well defined frequencies are observed and the associated spectrum strongly evolves during the growth process. The instability complex shape underlines the coexistence of different phenomena that could interact and give regular or chaotic oscillations. The instability time evolution reveals drastic and sudden changes in shape and frequency that could not be easily correlated to dust particle size and density. A detailed analysis of the instability main characteristics is performed in order to underline and bring to the fore the complex phenomena that could arise in a plasma containing changing dust particles.

\section{EXPERIMENTAL BASIS}

\section{A. Experimental set-up}

The work presented here is performed in the PKENefedov chamber designed for microgravity experiments [34]. The experimental set-up consists in a parallel plate rf discharge where an argon plasma $(0.2-2 \mathrm{mbar})$ is created in push-pull excitation mode $(0-4 \mathrm{~W})$. The electrodes are separated by $3 \mathrm{~cm}$ and their diameter is 4 $\mathrm{cm}$. The dust cloud is illuminated by a thin laser sheet perpendicular to the electrodes and the scattered light is recorded at $90^{\circ}$ with two standard CCD cameras at 25 images per second. In the first stages of growth, dust particles are very small and the scattered light is maxi- 
mum in the laser direction. Consequently, a third camera is watching the cloud with an angle lying approximately between $20^{\circ}$ and $30^{\circ}$ with respect to the incident laser direction. Instabilities are characterized by two different diagnostics. First, the time evolution of the amplitude of the discharge current fundamental harmonic is recorded. This electrical measurement is representative of global changes in plasma properties especially concerning the electron density. The second diagnostic is based on spatially resolved optical measurements. Five optical fibers are horizontally aligned ( $5 \mathrm{~mm}$ in between each fiber) and record the total plasma light with a spatial resolution of about $3 \mathrm{~mm}$. This diagnostic gives local measurements that are of interest to detect any plasma motion or localized changes in order to better understand the integrated electrical measurements.

\section{B. Dust particle growth}

Dust particles are grown by sputtering a polymer layer deposited on the electrodes and coming from previously injected dust particles $(3.4 \mu \mathrm{m}$, melamine formaldehyde). Typical working pressure is around 1.6 mbar and $\mathrm{rf}$ power is about $2.8 \mathrm{~W}$. With these parameters dust particles start to be detected by the cameras approximately $1 \mathrm{~min}$ after the plasma ignition. A more complete description of the experimental set-up and some results concerning dust particle growth are given in [9]. The growth process leads to various shapes of the dust cloud (few layers, domelike shape, 3D dense cloud) due to the fact that we do not control precisely size and density of grown dust particles. Indeed, the growth process seems to be highly sensitive to gas purity. This effect is amplified by the fact that experiments are performed at static pressure (no gas flow). At least one hour of pumping between each experiment is needed to eliminate species formed during the previous run and/or coming from the outgassing of the walls and/or from the sputtered matter. This behavior is well observed on successive runs by using emission spectroscopy. Impurities like $\mathrm{N}_{2}$ and $\mathrm{OH}$ are observed and their effect on dust particle formation is under investigations. Until now it is not clear if their presence prevents the sputtering or if the sputtering occurs but the growth cannot takes place. This effect of the gas purity has been previously reported in similar experiments [8]. Possible precursors like carbon molecules $\mathrm{C}_{2}, \mathrm{CN}$ and $\mathrm{CH}$ appear and the $\mathrm{C}_{2}$ molecule seems to be a good indicator of dust formation [35].

A very high dust density is obtained when base pressure before an experiment is sufficiently low (few $10^{-6}$ mbar). A base pressure of few $10^{-5}$ mbar drastically reduces the amount of grown dust particles. This base pressure dependence is even more drastic on the instability appearance. Indeed, in our experimental conditions, they are observed only if the base pressure is lower than $4 \times 10^{-6}$ mbar. Consequently, unstable conditions depend on gas purity and/or on dust particle density.

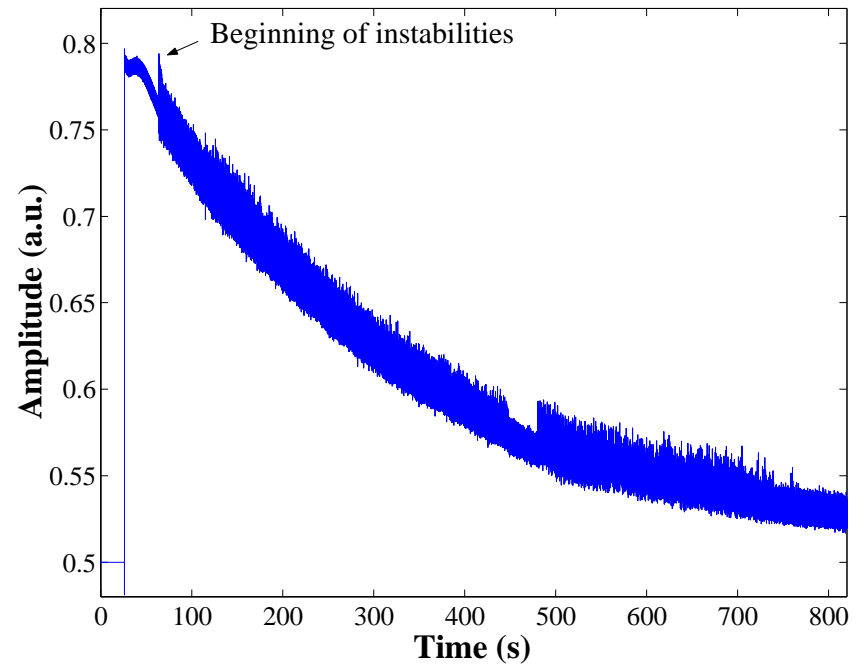

FIG. 1: (Color online). Time evolution of the amplitude of the discharge current fundamental harmonic during dust particle growth. Instabilities appear around $40 \mathrm{~s}$ after plasma ignition.

\section{DUST PARTICLE GROWTH INSTABILITIES}

Dust particle growth instabilities (DPGI) typically appear few tens of seconds after plasma ignition. Their appearance is well observed on both electrical and optical measurements $[36,37]$. Due to their low frequency and strong amplitude, DPGI beginning is also well detected by the naked eye looking at the plasma glow.

When dust particles are growing in a plasma, the amplitude of discharge current fundamental harmonic is decreasing due to the electron attachment on dust particle surface. This decrease is well observed in Fig. 1 from around $20 \mathrm{~s}$ (plasma ignition) to $800 \mathrm{~s}$. Appearance of DPGI is also clearly evidenced by an increase of the fluctuation amplitude becoming a real oscillation. DPGI can last several minutes (more than 12 minutes in Fig. 1) and different regimes are evidenced. Their beginning instant is slightly changing from one experiment to another due to differences in dust particle density which is strongly dependent on gas purity. Nevertheless, statistics performed on several experiments allow to deduce some general behavior. For exemple, the pressure dependence is shown in Fig. 2. An decreasing exponential variation of DPGI appearance time as a function of pressure is observed: the higher the pressure, the shorter the appearance time. This variation underlines the relation between the instability appearance and dust particle density. Indeed, DPGI are observed only when a huge dust particle density is obtained. From our previous experiments [35], we define the treshold pressure permitting dust particle growth around 1.2 mbar. At higher pressures, a shorter delay before formation of a dense cloud is measured. This delay is well evidenced in Fig. 2. At 1.4 mbar, nearly 2 minutes are necessary to initiate DPGI while only $40 \mathrm{~s}$ are required at 1.8 mbar. Two possibilities can explain this delay at low pressures: either a longer time is required to attain the critical dust density necessary to initiate DPGI or the dust density is lower and DPGI beginning needs bigger dust particles (ie longer time). These ex- 


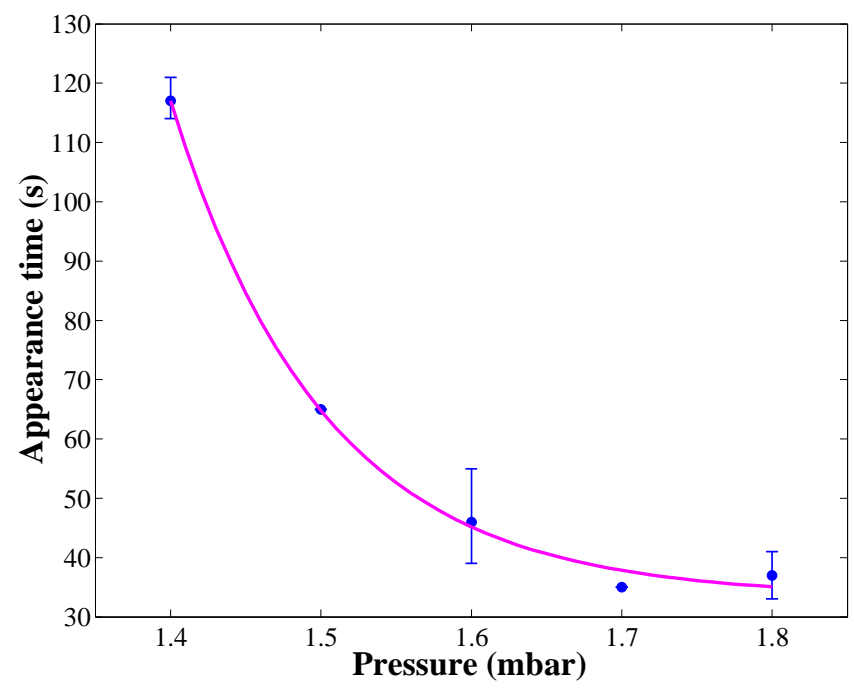

FIG. 2: (Color online). Instability appearance time as a function of argon pressure.

planations are consistent with another observation: the lower the rf power, the longer the appearance time.

\section{PHASE IDENTIFICATION: ELECTRICAL AND OPTICAL MEASUREMENTS}

As can be seen in Fig. 1, different regimes characterize DPGI. These different phases are easily brought to light by performing the same electrical measurements but in ac mode in order to improve oscilloscope vertical resolution. The resulting curve is presented in Fig. 3.

DPGI beginning is detected around $40 \mathrm{~s}$ and clear phases, numbered from 1 to 7 , are observed. These different phases are better evidenced by performing Fourier analysis of electrical signals. A typical spectrogram is given in Fig. 4. In order to emphasize small ordered domains, spectrogram intensity has been normalized in-

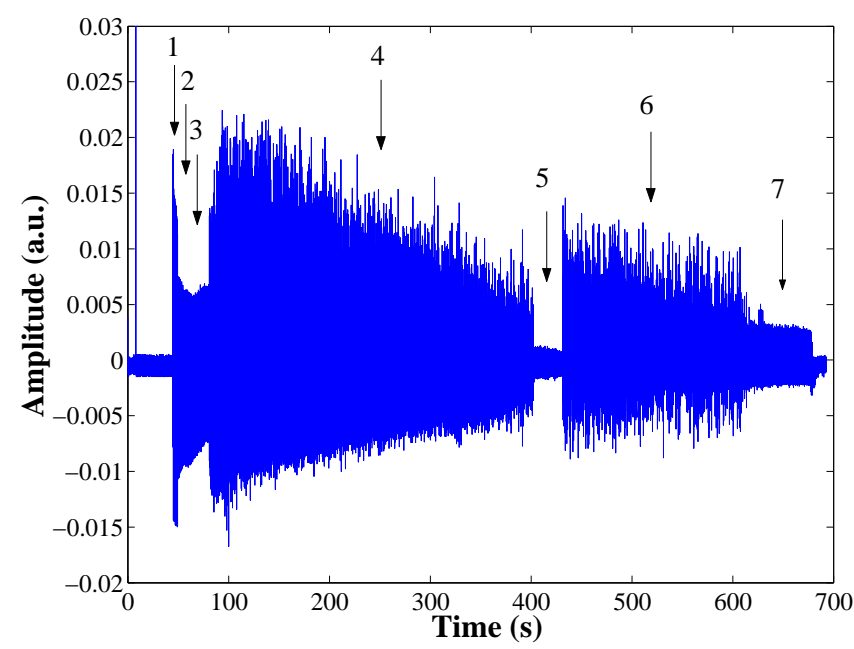

FIG. 3: (Color online). Time evolution of the amplitude of the discharge current fundamental harmonic during dust particle growth (ac component). Instabilities appear around $40 \mathrm{~s}$ after plasma ignition. Successive phases are numbered from 1 to 7 .

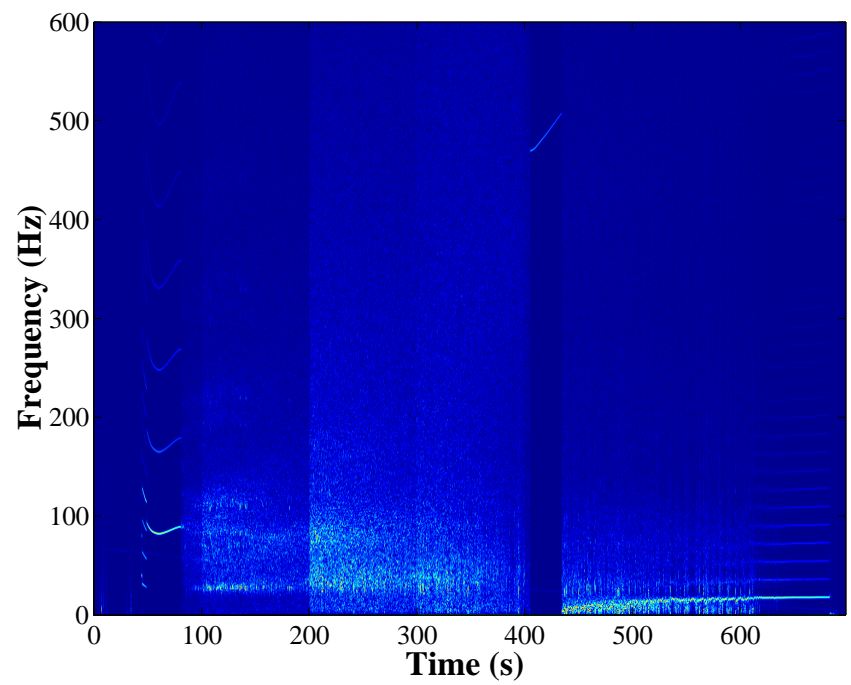

FIG. 4: (Color online). Spectrogram of electrical measurements corresponding to Fig. 3 and describing frequency evolution of DPGI as a function of time.

side each $100 \mathrm{~s}$ range (from 0 to $100 \mathrm{~s}$ intensity has been normalized to its maximum value inside this time domain and so on). Same Fourier analysis performed on spatially resolved optical measurements shows roughly the same spectrogram features and phases. From Figs. 3 and 4 we can identify seven different regimes (a precise analysis of these different phases will be presented in following sections):

- Three ordered phases P1, P2, P3 (from $\simeq 40 \mathrm{~s}$ to $\simeq 80 \mathrm{~s})$.

- Chaotic phase $\mathrm{P} 4$ (from $\simeq 80 \mathrm{~s}$ to $\simeq 405 \mathrm{~s})$.

- High frequency phase P5 (from $\simeq 405 \mathrm{~s}$ to $\simeq 435 \mathrm{~s}$ ).

- Chaotic phase becoming more and more regular P6 (from $\simeq 435 \mathrm{~s}$ to $\simeq 600 \mathrm{~s})$.

- Regular oscillation phase P7 (from $\simeq 600 \mathrm{~s}$ to $\simeq 680 \mathrm{~s})$

All these phases are observed on both electrical and optical measurements confirming their real correlation to an unstable state of the plasma-dust particle system. The global scheme of these instabilities is different from the one describe in $[7,8]$ where a filamentary mode (that could be related to our chaotic regime) is followed by a regular phase (great void mode). In our experiment, instabilities begin with regular oscillations (P1, P2, P3) followed by a long chaotic regime (P4). This chaotic phase (P4) suddenly ends with a high frequency phase $(\mathrm{P} 5)$ and starts again (P6) becoming more and more ordered. Finally, the system reaches a regular phase (P7) that can be sustained for a long time (in the exemple given here the plasma has been switched off at $680 \mathrm{~s}$ ).

\section{A. First three ordered phases}

DPGI begin with a succession of three ordered phases separated by clear transitions (Fig. 5). P1 and P2 phases are short and are not detected in all experiments while P3 phase lasts longer and is regularly observed. The 


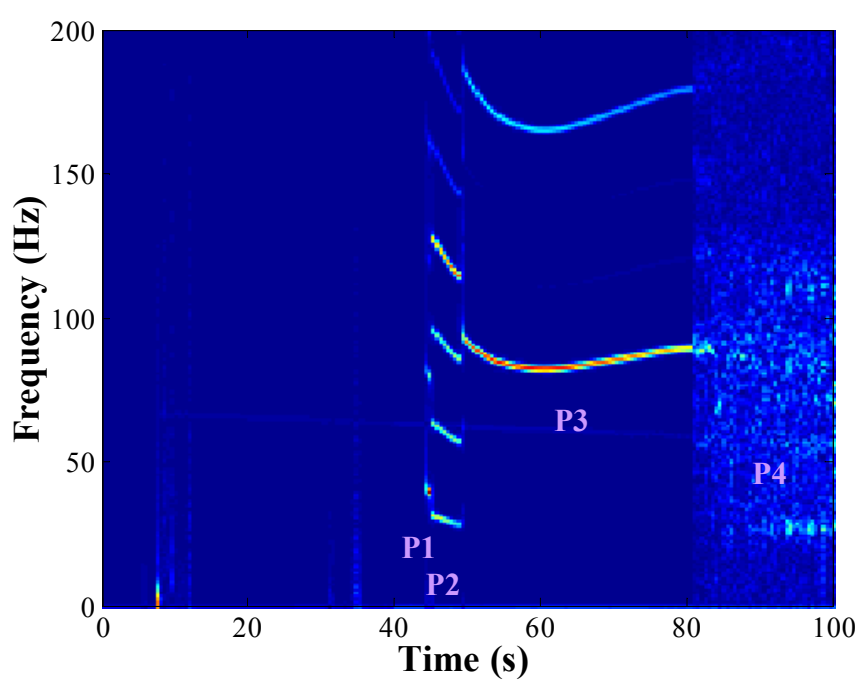

FIG. 5: (Color online). First three ordered phases of DPGI and transition to a chaotic regime.

three phases are well separated and evolve as a function of time. To explain the observed transitions between phases, it is necessary to also analyse the corresponding time series. As an example, Fig. 6 represents an electrical measurement obtained with a very short P2 phase in order to illustrate on the same figure P1-P2 and P2-P3 transitions. In Fig. 6, P1 phase is characterized by wide separated peaks with a mean frequency of about $40 \mathrm{~Hz}$. The transition from P1 to P2 corresponds to the growth of two small peaks between these higher amplitude patterns. The Fourier analysis traduces this change by a frequency decrease from about $40 \mathrm{~Hz}$ in $\mathrm{P} 1$ to about $30 \mathrm{~Hz}$ in $\mathrm{P} 2$. The small peaks continue to grow (P2 phase) and the higher amplitude ones decrease. Finally, all peaks reach the same amplitude characterizing P3 phase. The frequency of $\mathrm{P} 3$ phase is then approximately three times P2 frequency (around $94 \mathrm{~Hz}$ ). P3 phase is robust (nearly always observed in our experiments) and lasts a sufficiently long time to evolve with dust particle growth (Fig. 5). Its frequency time evolution is always the same, it decreases,

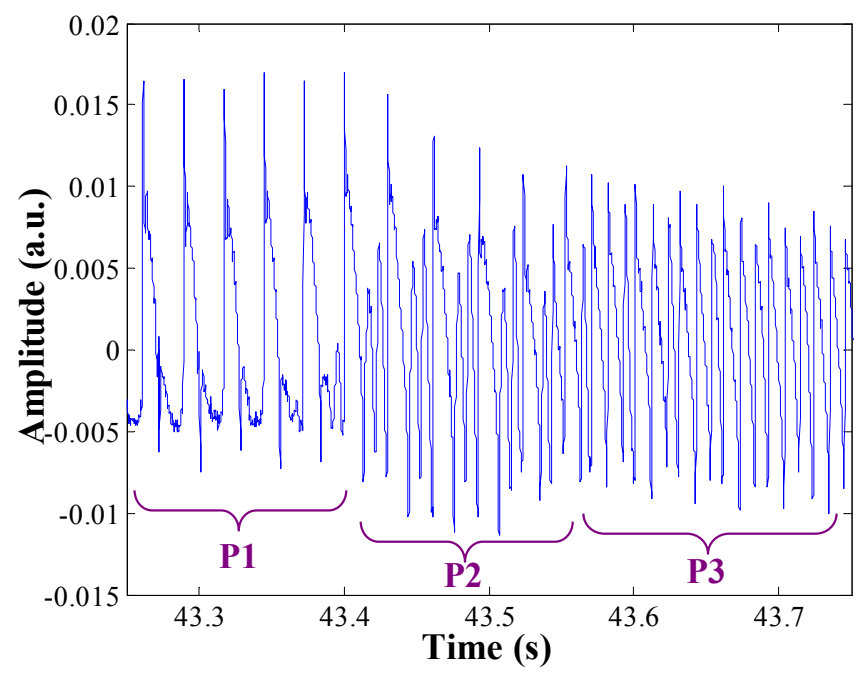

FIG. 6: (Color online). Transitions between the first three ordered phases observed on electrical measurements.

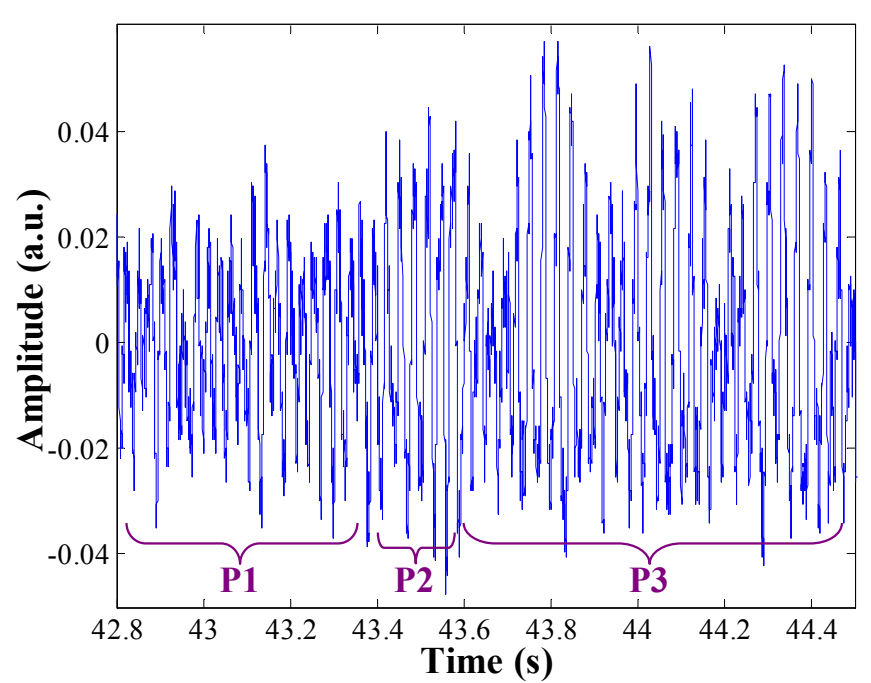

FIG. 7: (Color online). Transitions between the first three ordered phases observed on optical measurements performed near the plasma edge.

reaches a minimum value and then slightly increases until DPGI enter in the chaotic regime. Furthermore, the frequency range of $\mathrm{P} 3$ phase seems to be linearly dependent on the phase duration: the higher the frequency, the shorter the time duration. The time evolution of these three ordered phases is better observed on the frequency harmonics appearing due to the nearly sawtooth shape of electrical signals (Fig. 6).

Optical measurements recording the plasma light (integrated on all wavelengths) at different positions, show also three ordered phases (Fig. 7). Nevertheless, these P1, P2 and P3 phases have different characteristics than the ones observed on electrical measurements. Concerning the oscillation shape, the transition between P1 and P2 phases is here revealed by an increase of oscillation amplitude (Fig. 7). P3 phase is strongly marked by a clear amplitude modulation which is better evidenced near the plasma edge than in the plasma center. Concerning the oscillation frequency, some small discrepancies with electrical measurements are also observed, especially on P3 phase (Figs. 5 and 8). Indeed, P1 and P2 phases are similar but when DPGI enter in P3 phase, the typical frequency is not tripled, like in electrical measurements, but remains around the same value than during P2 phase. Furthermore, optical measurements of this P3 phase are slightly different depending on the observed plasma region. The signal recorded by the optical fiber watching the plasma center (Figs. 8(a) and 8(c)) is not exactly the same than the one recorded near the plasma edge (Figs. 8(b) and 8(d)). Indeed, the central fiber gives a main frequency around $26 \mathrm{~Hz}$ and a small amplitude component around $31 \mathrm{~Hz}$ (Fig. 8(c)). The near plasma edge fiber gives same frequencies but also additional ones: one in between 26 and $31 \mathrm{~Hz}$ and another one around 3 $\mathrm{Hz}$ (Fig. 8(d)) that corresponds to the strong modulation observed in Fig. 7. These observations underline that some spatial considerations must be made to interpret this P3 phase. The first local indication concerns the frequency three times lower than for electrical measurements. This point seems to signify that a phenomenon 

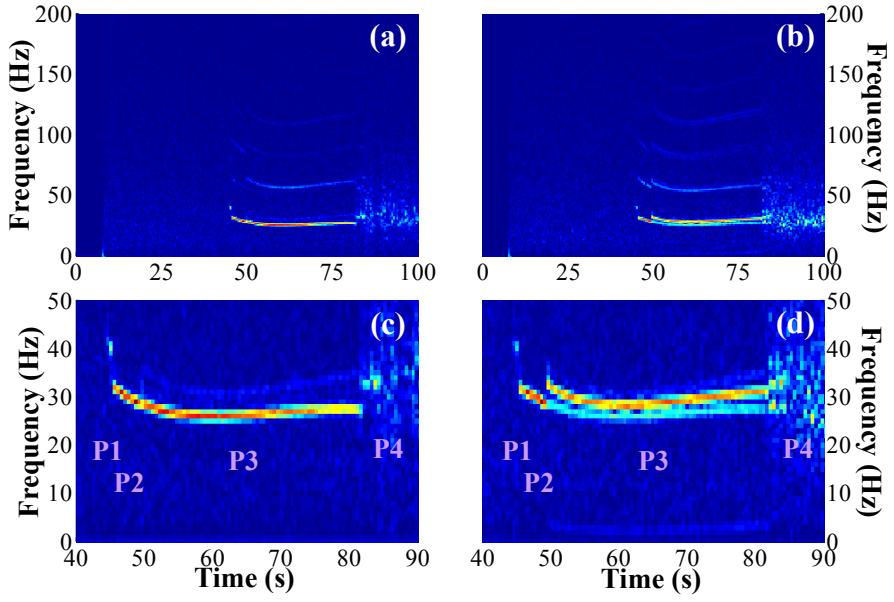

FIG. 8: (Color online). Spectrogram of optical measurements recorded (a) in the plasma center, (b) near the plasma edge. (c) zoom of (a), (d) zoom of (b).

oscillating at around $94 \mathrm{~Hz}$ affects the plasma, and consequently the electrical measurement, but is detected only one time out of three by the optical fibers. One possible explanation is that a plasma modification either appears successively in different places, or moves and comes back in front of an optical fiber with a frequency of about 30 $\mathrm{Hz}$. This plasma modification appears successively in distinct locations of the plasma because it is not detected at $94 \mathrm{~Hz}$ by any optical fiber looking at the plasma center or edge. Investigations concerning this hypothesis are currently underway using a high speed camera. The second local indication giving credit to this hypothesis, is the difference between observations given by each optical fiber. Indeed, even if their Fourier spectrum is nearly similar and shows only small discrepancies, the recorded time series bring more information. Optical measurements performed by the near plasma edge fiber is in phase opposition with ones in the center. This behavior, coupled with the fact that a stronger modulation is observed near the plasma edge, could confirm a possible motion, or appearance in different places, of a modified plasma region.

\section{B. Chaotic regime}

After three different ordered phases, DPGI enter in a chaotic regime $\mathrm{P} 4$ (Fig. 5 after $80 \mathrm{~s}$ ) with a strong increase in DPGI amplitude (Fig. 3). Then, the amplitude slowly decreases during the whole phase. P4 is also characterized by structured oscillations appearing in a transient manner. These structured regions are identified by some bright spots on electrical and optical spectrograms. On time series they appear as bursts of order. In Fig. 9(a), electrical signals reveal a clear transition between the P3 phase and the chaotic regime. This regime change is indicated by an arrow and a burst of order is encircled. A zoom of this burst of order is shown in Fig. 9(b). During the chaotic regime, structured oscillations in electrical signals always appear following a three peak structure (see for exemple between $94 \mathrm{~s}$ and $94.04 \mathrm{~s}$ in Fig. 9 (b)) that could be related to the three peaks observed
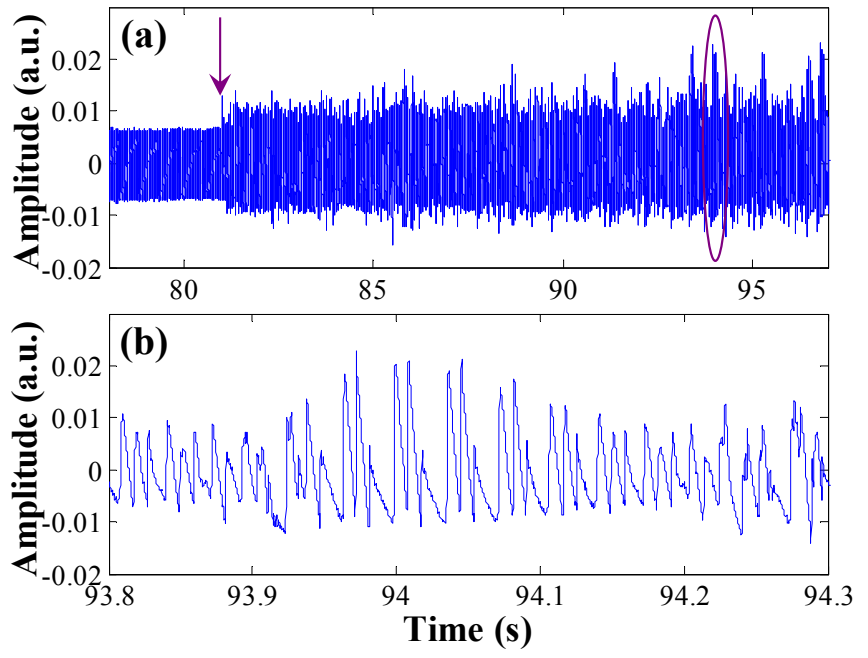

FIG. 9: (Color online). Electrical measurements: (a) transition between P3 and P4 phases and encircled burst of order, (b) zoom of the encircled part of Fig. 9(a).

in P2 or P3 phases. Indeed, it could be a reemergence of these phases during the chaotic regime. To compare our observations with the filamentary mode $[7,8]$, a Fourier spectrum of the whole chaotic regime (from 80 to 405 s) has been performed in Fig. 10. For electrical signals (Fig. 10(a)), a noisy main frequency around $28 \mathrm{~Hz}$ is observed and is equal to the frequency at the end of the P2 phase, confirming the possible reemergence of this phase. This frequency corresponds to the occurence frequency of the three peak structure regularly appearing during the chaotic regime (Fig. 9(b)). Due to these structures, multiple frequencies $(54 \mathrm{~Hz}, 83 \mathrm{~Hz}$ and $107 \mathrm{~Hz}$ corresponding to the frequency between two successive peaks of one single three peak structure) are also detected in Fig. 10(a). For optical measurements (Fig. 10(b)), differences with electrical ones are the same than in the P3 phase: one optical oscillation corresponds to a three peak structure and consequently only the $28 \mathrm{~Hz}$ frequency appears (Fig. 10(b)). These spectra are very similar to the one obtained for the filamentary mode [8] even if in our experiments the presence of more ordered domains is observed. Thus, we can surmise that the chaotic regime we observe could be similar to this filamentary mode.

\section{High frequency phase}

In some experiments, the chaotic regime is suddenly interrupted by a strong frequency change (at $405 \mathrm{~s}$ in Figs. 3 and 4 ). Indeed, this phase P5 is not always observed but when it is present its general characteristics are nearly always the same. This new phase appears after a continuous decrease of $\mathrm{P} 4$ phase amplitude and is defined by a radical change in DPGI amplitude and frequency. Indeed, DPGI turn into a low amplitude and high frequency (around $500 \mathrm{~Hz}$ ) oscillation as shown in Fig. 11. This change happens suddenly when no fast modifications in dust particle size and density are expected. Furthermore, the phase frequency increases with time. In Fig. 4, this increase is nearly linear but non linear behaviors have also been observed in certain cases. 

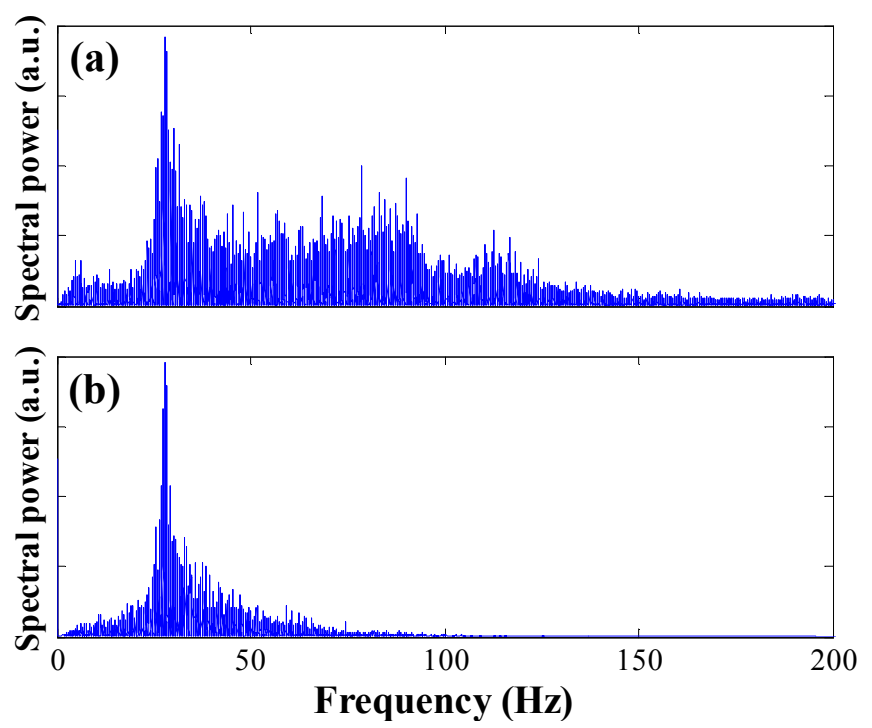

FIG. 10: (Color online). Fourier spectrum of the first chaotic regime on (a) electrical measurements, (b) central optical fiber measurements.

As previously mentionned, this phase is not always observed which means relatively precise conditions must be fulfilled for its existence. This effect is well confirmed in the experiment described in Fig. 12 where this phase is interrupted before starting again. Small modifications in plasma or dust particle properties can easily turn the system from the high frequency phase to the chaotic one. Furthermore, this P5 phase can also be transformed in regular oscillations as shown in Fig. 12 at $\mathrm{t}=294 \mathrm{~s}$. From these experimental results it can be assumed that this high frequency phase is a particular case of DPGI, obtained in a tight set of parameters and that can be easily turned in ordered or chaotic regimes.
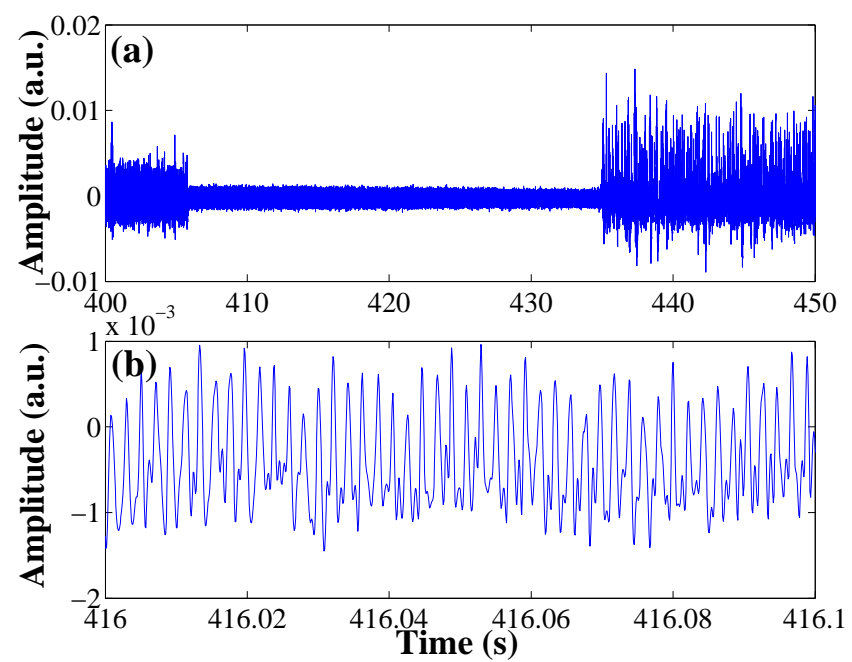

FIG. 11: (Color online). (a) Appearance of the high frequency phase in between two chaotic regimes, (b) zoom of (a).

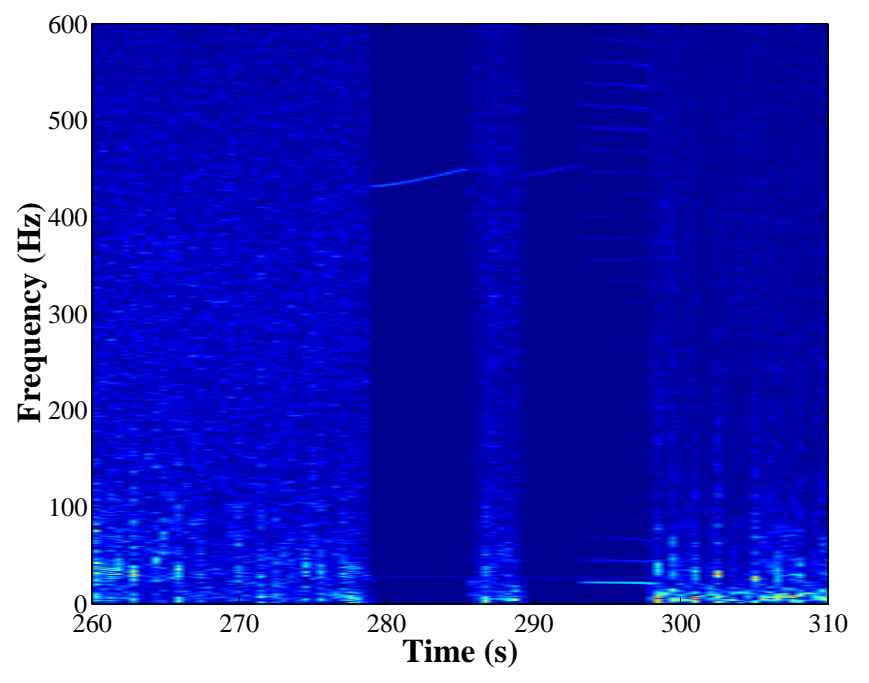

FIG. 12: (Color online). Spectrogram of a particular case of the high frequency phase. This phase is interrupted by a chaotic regime and is transformed in regular oscillations just before turning again in a chaotic regime.

\section{Second chaotic regime}

After the high frequency phase, a second chaotic regime is usually observed. Two different types of this P6 phase are observed. The most usual is presented in Fig. 4 between 435 and $600 \mathrm{~s}$. It corresponds to a phase similar to $\mathrm{P} 4$ but with more and more ordered regions. Figure 4 clearly shows that DPGI tend to stabilize to regular oscillations $(\simeq 600 \mathrm{~s})$. This transition appears as a small and continuous increase in DPGI frequency. Nevertheless, some experiments show a slightly different behavior as in Fig. 13. Instead of slowly increasing, the frequency decreases and enters (still decreasing) in a regular oscillation phase. This behavior corresponds to void rotation in a horizontal plane which is clearly evidenced thanks to CCD camera images. The corresponding spectrogram is nearly similar to the one obtained in [8] and

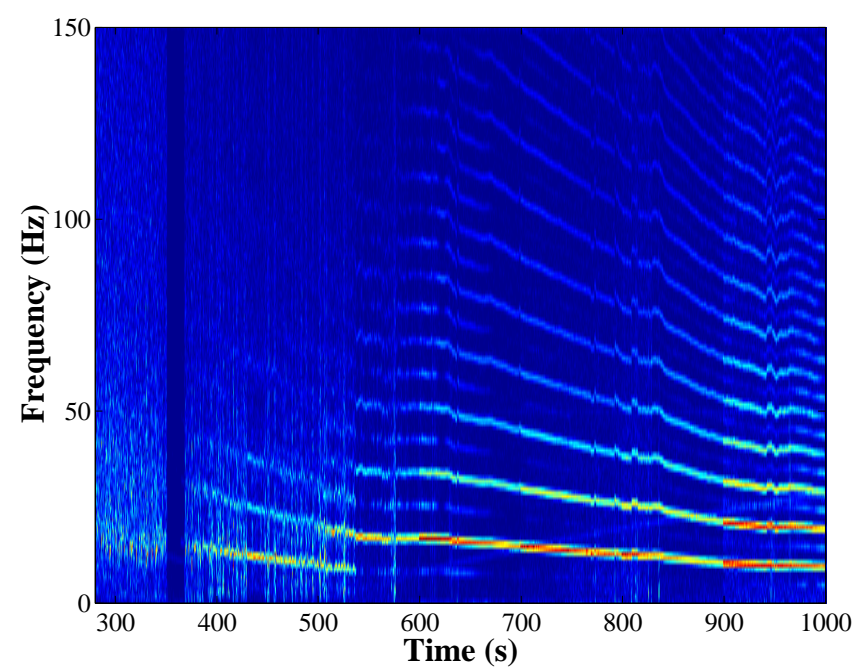

FIG. 13: (Color online). Spectrogram of a particular case of the second chaotic regime corresponding to void rotation in a horizontal plane. 


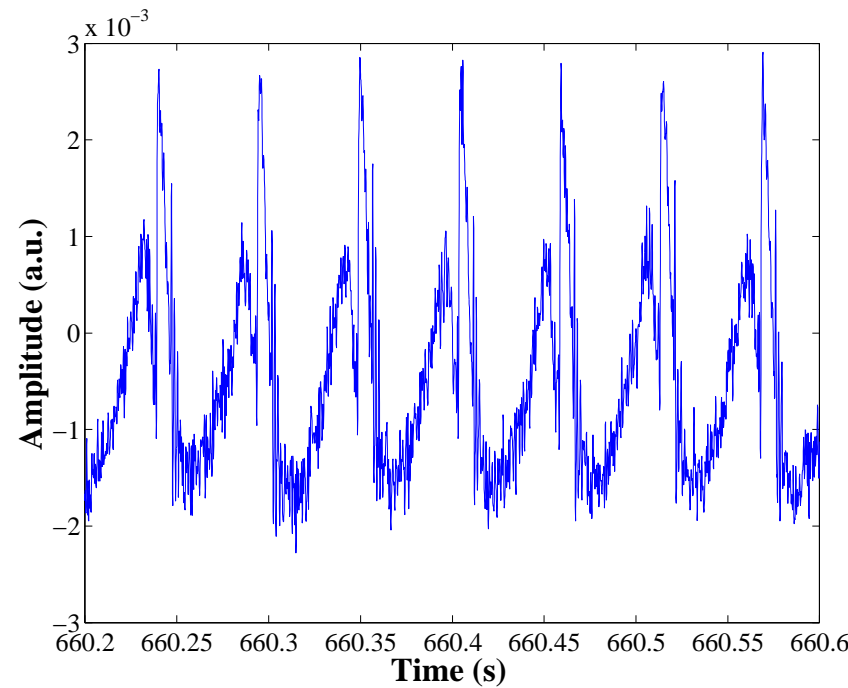

FIG. 14: (Color online). Electrical measurements corresponding to the final regular oscillation phase.

related to the great void mode.

\section{E. Final regular oscillation phase}

The final step of DPGI often corresponds to a regular oscillation phase characterized by a spectrogram with a typical frequency around $18 \mathrm{~Hz}$ (Fig. 4) and its harmonics. The corresponding electrical signal is shown in Fig. 14. The signal shape is in particular marked by a sharp peak appearing at each period. This shape is similar to what is obtained during the "heartbeat" instability $[23,38]$ which corresponds to regular contractions and expansions of the void size. At this stage of DPGI, this regular oscillation phase can last for minutes and is robust, meaning that once it is set up it does not turn in another regime. Optical measurements performed in the plasma center show an evolution similar to the electrical one except near the sharp peak region as observed in [36]. Furthermore, near edge optical fiber signal is in phase opposition with the central one (as in [36]) emphasizing the similarity with the heartbeat instability.

\section{DISCUSSION AND CONCLUSIONS}

In this paper, self-excited instabilities induced by the formation of submicron dust particles are investigated. These instabilities appear when a sufficiently high dust particle density is reached. Instability frequency and shape change during the growth process and direct correlation with dust particle size and density cannot be easily deduced. Indeed, effects of both parameters on discharge behavior are difficult to separate: a high density of small particles can have roughly the same effect than a lower density of bigger particles, assuming that the total collection surface is identical and consequently the same amount of electrons can be captured. The only way to conclude on this point is to measure dust particle size and density but most commonly used in-situ diagnostics cannot be implemented on our experimental setup. Furthermore, an easy systematic collection of dust particles coming from one and only one experiment is not possible in our reactor. DPGI reproducibility can be obtained only with very low base pressures not reached in case of regular reactor opening. By comparing Fig. 1 with electrical characteristics obtained in silane based discharges [39] we can speculate that DPGI occur during the "coalescence phase" and last during the so-called "surface deposition phase". These phases [40] are characterized by a significant amount of negatively charged dust particles strongly impacting on plasma stability. This comparison with dust particle growth process in silane based chemistry must be taken carefully because the analogy has not been strictly proved yet. From visual observations we know that when DPGI occur, dust particles are not detected yet by our video system and are certainly smaller than $100 \mathrm{~nm}$. When instabilities stop dust particle size could be of few hundreds of nanometers. The instability development follows a well-defined succession of phases that can be ordered or chaotic. At least seven different regimes have been evidenced and their occurence is strongly dependent on plasma and dust particle properties. Complex transitions between different ordered phases or between ordered and chaotic ones have been evidenced and underline the nonlinear behavior of these instabilities. Two observed phases seem to correspond to the ones reported in previous papers $[7,8]$. These phases, called the filamentary mode and the great void mode, can be a part of a more general behavior described in this paper. The new phases described here underline the complexity of phenomena behind observations and their high sensitivity to plasma and dust particle properties. Indeed, the complete scheme with seven phases can be observed in very specific experimental conditions usually encountered in this work. Striations (in our case, enhanced ionization regions appearing as bright spots or filaments) seem to be responsible for the different observed phases. The various regimes could be related to the way striations appear. Indeed, we can surmise that the ordered regimes could be striations appearing periodically and nearly at the same places. Following this hypothesis, the often observed three peak structure can correspond to three different striations. By analogy, chaotic regimes can be due to randomly appearing (in time and in space) striations. Optical measurements performed in different plasma positions bring to the fore that these instabilities need to be analyzed with spatial considerations. Differences with works reported in $[7,8]$ can be the consequence of different plasma and/or dust particle properties, geometrical considerations or gas flow. Furthermore, our observations show that void formation is not a consequence of these instabilities as proposed in [24]. The void can be formed with or without instabilities preceding its appearance. In some conditions, the void is formed before instability beginning. In this case, the instability evolution scheme does not describe all the phases presented here. Similarities between the final regular oscillation phase and the heartbeat instability concerning the void region have been observed. It could indicate that dust particle growth instabilities and the heartbeat instability could be different aspect of the same physical phenomena. 


\section{Acknowledgments}

The PKE-Nefedov chamber has been made available by the Max-Planck-Institute for Extraterrestrial Physics, Germany, under the funding of DLR/BMBF under grants No.50WM9852. The authors would like to thank S. Dozias for electronic support, J. Mathias for optical support and Y. Tessier for experimental support. This work was supported by CNES under contract 793/2000/CNES/8344.
[1] A. Bouchoule, A. Plain, L. Boufendi, J.-P. Blondeau, and C. Laure, J. Appl. Phys. 70, 1991 (1991).

[2] Y. Watanabe, M. Shiratani, Y. Kubo, I. Ogawa, and S. Ogi, Appl. Phys. Lett. 53, 1263 (1988).

[3] A. Howling, C. Hollenstein, and P. Paris, Appl. Phys. Lett. 59, 1409 (1991).

[4] G. Jellum and D. Graves, J. Appl. Phys. 67, 6490 (1990).

[5] W. Yoo and C. Steinbrüchel, J. Vac. Sci. Technol. A 11, 1258 (1993).

[6] B. Ganguly, A. Garscadden, J. Williams, and P. Haaland, J. Vac. Sci. Technol. A 11, 1119 (1993).

[7] G. Praburam and J. Goree, Phys. Plasmas 3, 1212 (1996).

[8] D. Samsonov and J. Goree, Phys. Rev. E 59, 1047 (1999).

[9] M. Mikikian, L. Boufendi, A. Bouchoule, H. Thomas, G. Morfill, A. Nefedov, V. Fortov, and the PKENefedov Team, New J. Phys. 5, 19 (2003).

[10] R. Roth, K. Spears, G. Stein, and G. Wong, Appl. Phys. Lett. 46, 253 (1985).

[11] G. Selwyn, J. Singh, and R. Bennett, J. Vac. Sci. Technol. A 7, 2758 (1989).

[12] L. Boufendi and A. Bouchoule, Plasma Sources Sci. Technol. 11, A211 (2002).

[13] P. Roca i Cabarrocas, P. Gay, and A. Hadjadj, J. Vac. Sci. Technol. A 14, 655 (1996).

[14] S. Tiwari, F. Rana, H. Hanafi, A. Hartstein, and E. Crabbé, Appl. Phys. Lett. 68, 1377 (1996).

[15] C. Szopa, G. Cernogora, L. Boufendi, J. Correia, and P. Coll, Planet. Space Sci. 54, 394 (2006).

[16] B. Walch, M. Horanyi, and S. Robertson, IEEE Trans. Plasma Sci. 22, 97 (1994).

[17] A. Melzer, T. Trottenberg, and A. Piel, Phys. Lett. A 191, 301 (1994).

[18] C. Arnas, M. Mikikian, and F. Doveil, Phys. Rev. E 60, 7420 (1999).

[19] L. Boufendi, J. Gaudin, S. Huet, G. Viera, and M. Dudemaine, Appl. Phys. Lett. 79, 4301 (2001).

[20] M. Cavarroc, M. Jouanny, K. Radouane, M. Mikikian, and L. Boufendi, J. Appl. Phys. 99, 064301 (2006).

[21] J. Dorier, C. Hollenstein, and A. Howling, J. Vac. Sci. Technol. A 13, 918 (1995).

[22] G. Morfill, H. Thomas, U. Konopka, H. Rothermel, M. Zuzic, A. Ivlev, and J. Goree, Phys. Rev. Lett. 83, 1598 (1999).

[23] M. Mikikian and L. Boufendi, Phys. Plasmas 11, 3733 (2004).

[24] J. Goree, G. Morfill, V. Tsytovich, and S. Vladimirov,
Phys. Rev. E 59, 7055 (1999).

[25] M. Akdim and W. Goedheer, Phys. Rev. E 65, 015401 (2001).

[26] V. Tsytovich, V. Vladimirov, G. Morfill, and J. Goree, Phys. Rev. E 63, 056609 (2001).

[27] G. Gozadinos, A. Ivlev, and J.-P. Boeuf, New J. Phys. 5, 32.1 (2003).

[28] K. Avinash, A. Bhattacharjee, and S. Hu, Phys. Rev. Lett. 90, 075001 (2003).

[29] C. Zafiu, A. Melzer, and A. Piel, Phys. Plasmas 10, 1278 (2003).

[30] A. Ivlev, S. Zhdanov, S. Khrapak, and G. Morfill, Phys. Rev. E 71, 016405 (2005).

[31] M. Kretschmer, S. Khrapak, S. Zhdanov, H. Thomas, G. E. Morfill, V. Fortov, A. Lipaev, V. Molotkov, A. Ivanov, and M. Turin, Phys. Rev. E 71, 056401 (2005).

[32] S. Vladimirov, V. Tsytovich, and G. Morfill, Phys. Plasmas 12, 052117 (2005).

[33] V. Land and W. Goedheer, New J. Phys. 8, 8 (2006).

[34] A. Nefedov, G. Morfill, V. Fortov, H. Thomas, H. Rothermel, T. Hagl, A. Ivlev, M. Zuzic, B. Klumov, A. Lipaev, et al., New J. Phys. 5, 33.1 (2003).

[35] M. Mikikian, L. Boufendi, and A. Bouchoule, in Proc. $30^{\text {th }}$ EPS Conf. on Contr. Fusion and Plasma Phys., St Petersburg, edited by R. Koch and S. Lebedev (2003), vol. 27A of ECA, pp. O-3.1B, URL http://epsppd. epfl.ch/StPetersburg/PDF/03_001B.PDF.

[36] M. Mikikian, M. Cavarroc, N. Chaumeix, and L. Boufendi, in Proc. of the $31^{\text {st }}$ EPS Conf. on Plasma Phys., London, edited by P. Norreys and H. Hutchinson (European Physical Society, Petit-Lancy, 2004), vol. 28G of ECA, pp. O-2.13, URL http://epsppd.epfl.ch/ London/pdf/02_13.pdf.

[37] M. Mikikian, M. Cavarroc, and L. Boufendi, in New Vistas in Dusty Plasmas, edited by L. Boufendi, M. Mikikian, and P. Shukla (2005), vol. 799 of AIP Conference Proceedings, p. 319.

[38] M. Mikikian, M. Cavarroc, and L. Boufendi, in New Vistas in Dusty Plasmas, edited by L. Boufendi, M. Mikikian, and P. Shukla (2005), vol. 799 of AIP Conference Proceedings, p. 323.

[39] M. Cavarroc, M. Mikikian, G. Perrier, and L. Boufendi, Appl. Phys. Lett. 89, 013107 (2006).

[40] A. Bouchoule, L. Boufendi, J. Hermann, A. Plain, T. Hbid, G. Kroesen, and W.W. Stoffels, Pure Appl. Chem. 68, 1121 (1996). 